\section{POSTER 513}

\section{The Incidence, Source, and Nature of Complaints Received in a Large, Urban EMS System}

\author{
Curka PA, Pepe PE, Zachariah BS, \\ Gray GD, Matsumoto $C$ \\ Baylor College of Medicine \\ City of Houston Emergency Medical Services \\ Houston, Texas, USA
}

Background: Emergency Medical Services system managers traditionally have measured quality using survival rates, response times, and citizen surveys. Another measure could be an analysis of complaints received. To date, however, published reports detailing the frequency, source, and nature of EMS complaints are lacking.

Purpose: To document the incidence, source, and reasons for all complaints and inquiries received by a large, municipal EMS program.

Methods: A retrospective review of all complaints and inquiries received during three consecutive years (1990-1992). All cases were then categorized by year, source, and nature of the complaint.

Results: In the three years, the EMS system responded to 416,892 incidents with nearly a half million patient contacts. Concurrently, 371 complaints and inquiries were received (frequency of <.09\%); 132 in 1990, 129 in 1991; and 110 in 1992. Two-thirds involved: 1) allegations of "rude or unprofessional conduct" $(34 \%) ; 2)$ "didn't take patient to the hospital" $(18 \%)$; and 3) "problems with medical treatment" (13\%). Only $1.6 \%(\mathrm{n}=6)$ were response time complaints. Other complaints ranged from lost/damaged property, wrong hospital, inappropriate billing and driving habits. The most common sources were patient families $(39 \%)$ and the patients themselves $(30 \%)$. Only $7.8 \%$ were from health care providers.

Conclusion: Reviews of complaints and inquiries provide a measure of EMS system performance, but also can reveal targets for quality improvement. For this system, this study suggests a future training focus on interpersonal skills and heightened sensitivities toward patients, bystanders, and family.

\section{POSTER 515}

\section{Analysis of the Need and Current Utilization of Prehospital Do-Not-Resuscitate Orders}

\author{
Isaacs ED, Mower WR, Rottman SJ \\ Emergency Medicine Center, UCLA School of Medicine \\ Los Angeles, California, USA
}

Purpose: Evaluate the need and utilization of prehospital "Do Not Resuscitate" (DNR) orders in an EMS system that lacks established DNR protocols.

Methods: Each base-station in the Los Angeles (LA) County EMS system was sampled during a random month of a 12month period. During each sample period, prehospital care encounters involving advanced-life-support paramedics were analyzed prospectively to determine if individual encounters could be eligible for DNR orders. Encounters were designated as eligible for DNR orders if the patient receive cardiopulmonary resuscitation (CPR) or was pronounced dead by prehospital care providers. The maximum need for DNR orders was defined to be the fractional percent of cases eligible for DNR out of the total number of prehospital evaluations. A DNR order was considered to have been utilized if any type of written advance medical directive was available for prehospital personnel at the time of medical intervention.

Setting and Participants: Patients undergoing LA County EMS prehospital evaluation.

Results: A total of 300 encounters were eligible for DNR orders out of 8,499 prehospital evaluations; thus DNR orders might have been useful in at most $3.53 \%( \pm 0.20 \%)$ of the prehospital care encounters. Written directives were utilized in only $9.35 \%$ of the encounters eligible for DNR orders, or $0.33 \%$ $( \pm 0.062 \%)$ of all encounters.

Conclusions: DNR orders may be useful in a very small minority of prehospital care encounters. However, written directives were infrequently available as a source of DNR instructions. In this setting, paramedics would need to make more than 300 evaluations for each directive encountered. Efforts to limit inappropriate prehospital resuscitations should focus on: 1) developing criteria to allow prehospital personnel to withhold CPR; and 2) increasing the use of standardized, written advance directives. 